TERRA. Revista de Desarrollo Local e-ISSN: 2386-9968

Número 6 (2020), 313-322

DOI 10.7203/terra.6.17074

IIDL - Instituto Interuniversitario de Desarrollo Local

\title{
Usando un enfoque del Hemisferio Sur para crear un gran destino de naturaleza en España: Maestrazgo-Els Ports
}

\author{
Ignacio Jiménez Pérez \\ Iniciativa Maestrazgo-Els Ports (Valencia, España) \\ i_jimenez_perez@yahoo.es
}

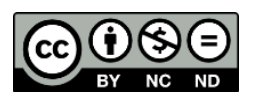

Esta obra se distribuye con la licencia Creative Commons Reconocimiento-NoComercial-SinObraDerivada 4.0 Internacional 


\section{SECCIÓN EXPERIENCIAS Y BUENAS PRÁCTICAS}

\section{Usando un enfoque del Hemisferio Sur para crear un gran destino de naturaleza en España: Maestrazgo-Els Ports}

Resumen: A partir de dos casos de éxito en la generación, organización y gestión de áreas naturales como espacios protegidos en Latinoamérica, se avanza hacia la traslación y adaptación del proceso de Producción de Naturaleza a un ejemplo europeo: el área interautonóminca del Maestrazgo-Els Ports en España. Las estrategias de comunicación y articulación para el desarrollo de esta iniciativa llevan en marcha desde un plano informal desde el año 2020, en un área histórico-cultural y natural que cuenta con diversas diferencias, tanto en positivo como en negativo, con los ejemplos latinoamericanos que sirven de guía. La iniciativa Maestrazgo-Els Ports está recién dando sus primeros pasos y en los próximos años veremos si el enfoque de Producción de Naturaleza desarrollado en el Hemisferio Sur puede ser aplicado con éxito en un paisaje rural español y el tipo de ajustes necesarios para que esto suceda.

Palabras clave: Producción de Naturaleza, ecoturismo, Parque Nacional Iberá, áreas protegidas, ecosistemas naturales, desarrollo territorial, Gran Reserva de Mata Atlántica, orgullo comunitario, sentido de pertenencia.

Recibido: 03 de abril de 2020

Devuelto para revisión: -

Aceptado: 04 de abril de 2020

Referencia / Citation:

Jiménez, I. (2020). Usando un enfoque del Hemisferio Sur para crear un gran destino de naturaleza en España: Maestrazgo-Els Ports. TERRA. Revista de Desarrollo Local, (6), 313-322. DOI 10.7203/terra.6.17074 


\section{LA CREACIÓN Y APLICACIÓN DEL CONCEPTO DE PRODUCCIÓN DE NATURALEZA EN ARGENTINA}

Entre los años 2005 y 2018 trabajé como coordinador de diferentes programas para la fundación The Conservation Land Trust o CLT. Esta fundación fue creada por el empresario textil y filántropo Douglas Tompkins con el propósito principal de crear grandes parques nacionales en Chile y Argentina, además de apoyar programas de conservación y restauración ecológica en su interior y entorno más cercano (Butler, 2016). El principal proyecto en el que participé dentro de CLT tenía como objetivo la compra, restauración ecológica y donación de 150,000 ha de antiguas estancias ganaderas para crear el Parque Nacional Iberá en el nordeste de Argentina (www.proyectoibera.org). Dicho parque nacional, unido a un parque provincial colindante de 550,000 ha acabaría constituyendo el mayor parque del país. En paralelo, el proyecto liderado por CLT incluía la reintroducción de seis especie de mamíferos localmente extintos (oso hormiguero gigante (Myrmecophaga tridactyla), venado de las pampas (Ozotoceros bezoarticus), tapir (Tapirus terrestres), pecarí de collar (Tajacu pecari), jaguar (Panthera onca) y nutria gigante (Pteronura brasiliensis)) y una gran ave (el guacamayo rojo (Ara chloroptera)) extinta a nivel nacional. En conjunto, se trata del mayor programa de reintroducción de fauna localmente extinta o rewilding del continente americano (Zamboni et al., 2017).

Un aspecto notable de este proyecto es que, cuando fue comunicado públicamente, la respuesta de la sociedad local, provincial (i.e. Corrientes) y nacional fue principalmente de desconfianza o desinterés. En el primer caso, no se creía que una fundación de origen extranjero fuera a donar 150,000 ha de propiedad privada a la nación argentina y se optó por crear explicaciones alternativas (p.ej. un complot imperialista para quedarse con las reservas de agua de la región). En el segundo caso, se admitían las intenciones conservacionistas de la fundación filantrópica pero se ponía en duda el valor de dicha donación. En esos casos se cuestionaba la importancia de la conservación frente al uso más aceptado de la producción.

Esta situación nos alentó a reformular la manera como comunicábamos nuestro proyecto y a crear el concepto de Producción de Naturaleza. Bajo este enfoque, los espacios protegidos (en este caso, el parque que estábamos por crear) que albergan ecosistemas completos con fauna abundante y fácil de ver (incluyendo las especies que estábamos reintroduciendo) sirven como base de una nueva economía local basada en gran medida en el ecoturismo, la cual permitía que las comunidades rurales tuvieran mejores oportunidades laborales y de desarrollo, a la vez que se fomentaba un sentido de orgullo e identidad propios (Figura 1).

En este caso nos sirvió mostrar como el pueblo más cercano a nuestras propiedades (Colonia Carlos Pellegrini) manifestaba mejores indicadores de empleo que otros pueblos de la región, gracias al desarrollo de una economía de servicios basada en el ecoturismo. Y podíamos exponer cómo esta economía se nutría de lo que producíamos en nuestros campos: paisajes naturales y fauna silvestre. Gracias al uso del término Producción de Naturaleza empezamos a comunicar el parque que estábamos por crear como una gran área productiva, en lugar de un área únicamente de conservación, como se veía anteriormente. Como resultado de esto, la región de Iberá se empezó a proyectar como un destino global de ecoturismo, lo que generó cambios importantes a múltiples escalas. En el ámbito local favoreció que varios alcaldes mostraran su interés en participar en el proyecto de un futuro parque nacional. En el ámbito provincial provocó la creación de un 
nuevo Ministerio de Turismo para poder gestionar el nuevo destino generado y la aprobación por ley del parque nacional. En el ámbito nacional implicó el uso por parte del gobierno federal del "Caso Iberá" como modelo de desarrollo a aplicar en otras regiones y la aprobación final del Parque Nacional Iberá (que pocas personas querían al principio del proyecto) por el parlamento argentino. En el ámbito global supuso la inclusión de Iberá en sucesivos listados de lugares recomendados a visitar, como sucedió con National Geographic, The New York Times y Lonely Planet.

Figura 1. Diagrama que muestra el concepto de Producción de Naturaleza creado por el Proyecto Iberá en Argentina

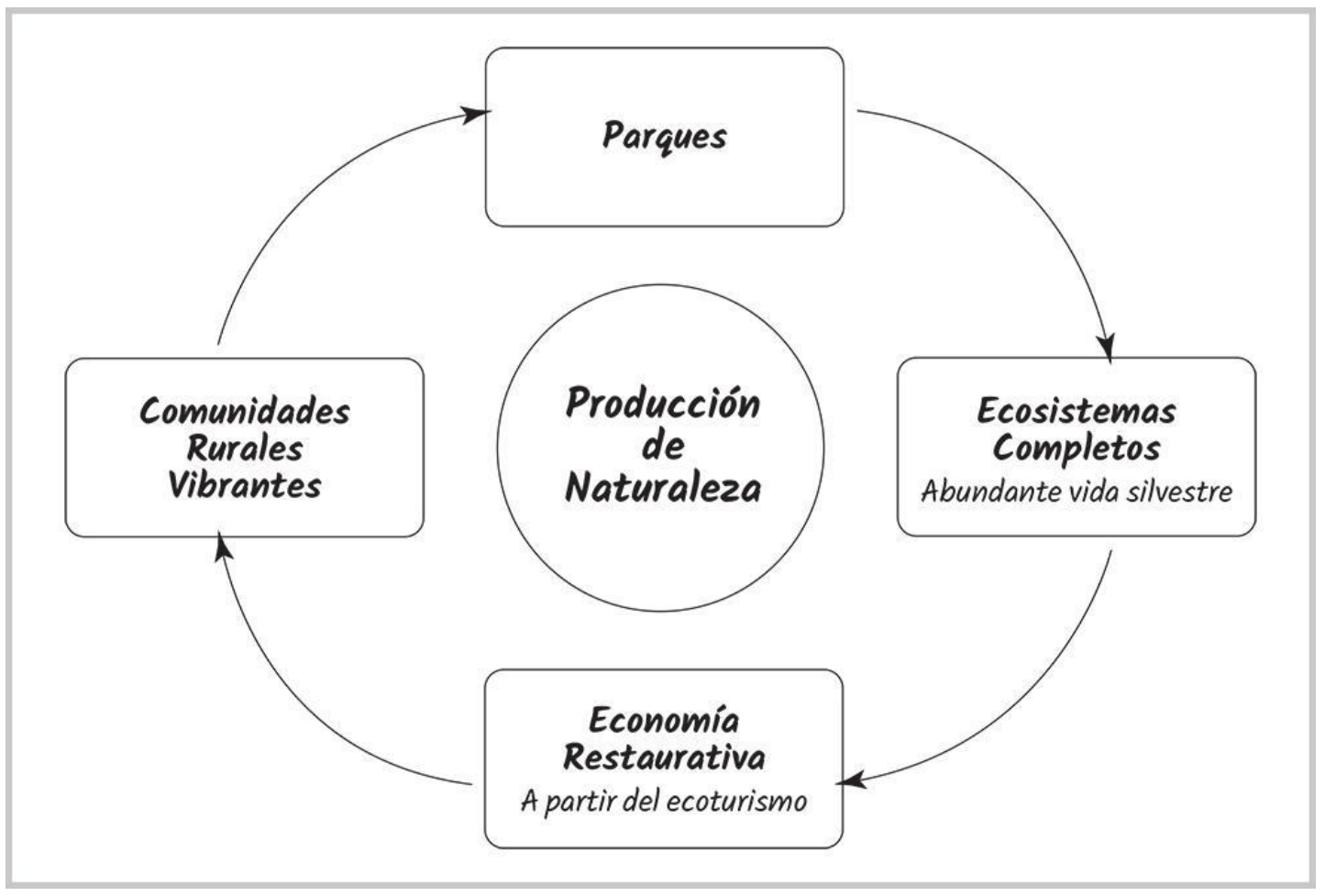

Fuente: Jiménez Pérez, 2018.

Luego, mediante esta experiencia descubrimos cómo el uso del término Producción de Naturaleza permitía mudar una postura de desconfianza o desinterés en relación a la conservación y restauración de un gran paisaje silvestre, para convertirla en entusiasmo y apoyo público. También durante esos años identificamos que esta estrategia dirigida a comunicar los ecosistemas naturales como generadores de empleo y desarrollo, ya estaba siendo empleada exitosamente y con diferentes matices en países tan diversos como Estados Unidos, Costa Rica, Namibia, Ruanda, India y Sudáfrica (Jiménez Pérez, 2018).

Gracias a nuestra propia experiencia en Iberá y a identificar procesos similares en otras regiones del mundo, descubrimos que la promoción de grandes espacios naturales como destinos ecoturísticos que generan empleo, desarrollo y orgullo, permitía generar un amplio apoyo público hacia su conservación y restauración. Es decir, estos espacios podían sean considerados como bienes valiosos por parte de amplios sectores de la sociedad, más allá de los grupos conservacionistas clásicos. A partir de este descubrimiento, la lógica basada en establecer un gran destino de naturaleza alrededor de los parques que estábamos creando permitió el establecimiento del Parque Nacional Impenetrable en la provincia del Chaco y la del Parque Nacional Patagonia en la provincia 
de Santa Cruz, con la complejidad añadida en este último caso de hablar de un destino binacional compartido con Chile que unía dos parques nacionales que usaban el mismo nombre de Patagonia. Para una versión más completa sobre la creación y uso del concepto de Producción de Naturaleza se puede consultar a Heinonen (2018) y Jiménez Pérez (2018).

\section{LA AMPLIACIÓN Y ADAPTACIÓN DEL ENFOQUE DE PRODUCCIÓN DE NATURALEZA HACIA BRASIL}

El siguiente paso fue aplicar esta misma lógica en un contexto diferente. En el año 2016 los dirigentes de la organización ambientalista brasileña Sociedade de Pesquisa em Vida Selvagem e Educação Ambiental o SPVS visitaron el Proyecto Iberá. La principal inquietud de estos dirigentes era que, después de 30 años de trabajo, sentían que no estaban logrando un consenso social alrededor del cuidado de las áreas protegidas y los ecosistemas naturales. En pocas palabras, sentían que a pesar de muchos años de esfuerzos, no estaban logrando resultados de alto impacto. Como resultado de esta visita, los representantes de SPVS se comenzaron a plantear cómo podían dar un "salto de escala" desde los proyectos pequeños y dispersos, hacia una iniciativa ambiciosa que generase un impacto mayor.

En el año 2017 varios de los miembros de CLT visitamos el área de trabajo en SPVS en el litoral del estado de Paraná para evaluar el potencial de esa región y comprender lo que estaba sucediendo en ella. Lo que descubrimos fue un grupo de organizaciones conservacionistas compuesto por ONG y organismos gubernamentales que transmitían una fuerte sensación de desánimo y que veían el área donde trabajaban como un mosaico de áreas protegidas separadas entre sí y con escaso apoyo público (Jiménez Pérez, 2018). A partir de este conocimiento, miembros del equipo de CLT en Argentina comenzamos a trabajar con SPVS y sus organizaciones aliadas para crear una nueva visión de la región, donde lo que se percibía como un mosaico de áreas protegidas más o menos dispersas e infravaloradas, podía ser percibido y comunicado como el mayor bloque continuo de Mata Atlántica del mundo. De repente comenzamos a hablar de más de dos millones de hectáreas continuas de bosques tropicales dentro de uno de los principales "hotspots" de biodiversidad mundial (Ribeiro et al., 2011). Esto nos permitió iniciar una campaña de comunicación destinada a presentar a esta gran extensión de bosque tropical -bautizada como la Gran Reserva de Mata Atlántica - como la gran oportunidad de desarrollo para las comunidades locales, muchas de ellas con los menores indicadores del Índice de Desarrollo Humano de sus respectivos estados (San Pablo, Paraná y Santa Catarina). Es decir, gracias al enfoque de Producción de Naturaleza comenzamos a convertir una narrativa de pérdida con baja adherencia pública (i.e. fragmentos separados de una selva con escasa utilidad práctica para las comunidades locales y sus respectivos estados), en un relato de una gran área natural que servía como motivo de orgullo ante el mundo y como gran oportunidad económica en áreas donde otras actividades tradicionales (agricultura a gran escala, ganadería o industria) no tenían perspectivas claras de generar desarrollo y empleo.

A partir del 2018 este proceso se intensificó y yo comencé a trabajar directamente con el equipo directivo de SPVS en este sentido. Este trabajo se centró en el uso de dos estrategias de trabajo simultáneas y complementarias, las cuales las denomino como de comunicación y articulación. La campaña de comunicación se basa en el establecimiento de la "marca" Gran Reserva Mata Atlántica como una gran área de producción de 
naturaleza, combinando aspectos de biodiversidad, áreas protegidas, cultura local y turismo dentro de una misma narrativa unificada. Y esta marca se comenzó a comunicar en el ámbito local, de los tres estados y nacional mediante redes sociales, videos, folletos, entrevistas en medios de comunicación y presentaciones públicas (ver http://grandereservamataatlantica.com.br/). En paralelo, el trabajo de articulación implicó decenas de conversaciones con múltiples actores locales donde presentábamos el concepto de Producción de Naturaleza aplicado a la Gran Reserva Mata Atlántica y escuchamos sus visiones, intereses, esperanzas y preocupaciones. Esto permitió poner a prueba y ajustar los mensajes de la campaña de comunicación, a la vez que se establecían vínculos personales de confianza y colaboración con múltiples líderes y grupos dispersos en un territorio tan amplio. De este modo, las visitas de articulación con encuentros personalizados combinadas con la creación y difusión de productos de comunicación de alta calidad se reforzaban entre sí, e iban generando un movimiento social apoyado por decenas de instituciones públicas (ayuntamientos y oficinas públicas estatales) y privadas (ONG y negocios). Más allá de sus diferentes intereses y culturas organizativas, estas instituciones compartían una misma visión de futuro centrada en un territorio amplio y en el concepto de Producción de Naturaleza. Al igual que sucedió con CLT en Argentina, el cambio desde un relato con connotaciones más bien negativas y exclusivamente ambiental a otro más esperanzador e integrador, permitió que SPVS y sus socios tuvieran acceso a políticos y tomadores de decisiones a los que anteriormente apenas podían llegar. Y esto permitió a su vez, que el concepto de la Gran Reserva Mata Atlántica y la conservación de los bosques de la región fueran incorporados por grupos que anteriormente no lo incluían en su agenda política e institucional. También como resultado de este proceso, en el año 2019 se publicó en Brasil la traducción al portugués del manual de producción de naturaleza que se había publicado en español el año previo (Jiménez Pérez, 2018) y esta publicación fue reflejada por medios de comunicación que trascendían el ámbito de lo ambiental. Finalmente, la experiencia desarrollada con SPVS en la Mata Atlántica permitió que la metodología basada en el uso conjunto de herramientas comunicación y articulación, fuera aplicada en otra ecorregión del país (Pantanal) con otra ONG ambientalista (Instituto Home Pantaneiro) para crear otro gran destino y área de producción de naturaleza bautizada como Alto Pantanal (www.altopantanal.com.br).

\section{LA EXPERIENCIA ESPAÑOLA: MAESTRAZGO-ELS PORTS}

El siguiente paso era ver si este enfoque podía ser aplicado en el contexto europeo. Para lograr esto en el año 2018 retorné a la Comunidad Valenciana, mi lugar de nacimiento y donde viví hasta los 24 años. Desde el principio España aparecía como el país de Europa con mayor potencial para aplicar el concepto de Producción de Naturaleza. Esto se basaba en los siguientes datos. En primer lugar, España es el país de Europa con mayor superficie de espacios incluidos dentro de Red Natura 2000, estando el $28 \%$ del área terrestre bajo esta categoría (European Environment Agency, 2018). En segundo lugar, el país destaca por servir como el principal refugio o uno de los principales del continente, para especies de fauna silvestre como el oso pardo, lobo, lince ibérico, buitre leonado y negro, águila imperial, águila perdicera, quebrantahuesos, ciervo común, rebeco pirenaico y avutarda, entre otras (Deinet et al., 2013). En tercer lugar, el país se encuentra entre los tres primeros destinos turísticos del mundo, con más de 80 millones de visitantes por año (INE, 2019). Finalmente, España tiene un serio problema de despoblamiento rural, lo que 
hace que se necesiten nuevas opciones económicas para pueblos que están perdiendo su población de manera constante.

El siguiente paso fue identificar una región dentro de España con potencial para convertirse en un gran destino de naturaleza similar a los creados en Argentina y Brasil o a los existentes en otros países (p.ej. Yellowstone en Estados Unidos, Galápagos en Ecuador, Serengueti en Tanzania, Etosha en Namibia o Kruger en Sudáfrica). Una región que podía cumplir con este requisito es el área fronteriza entre Aragón, Cataluña y la Comunidad Valenciana conocida como Maestrazgo-Els Ports (Figura 2). Esta región cuenta con atributos interesantes que le permiten convertirse tanto en un destino global de ecoturismo como en uno de los grandes paisajes europeos de conservación. Se trata de un área de unas 550.000 has con una notable continuidad ecológica y cultural ubicada en un paisaje de montaña con una densidad de 7,9 habitantes $/ \mathrm{km}^{2}$. Después de un año de realizar decenas entrevistas informales con habitantes de la región, pude confirmar un sentido de pertenencia compartido entre ellos. Esto hace que se sientan más cercanos a los habitantes de los pueblos vecinos situados en otras Comunidades Autónomas que a sus capitales provinciales, y todavía más que a sus capitales autonómicas. Es decir, un habitante de Morella (Castellón) se siente más cercano a otro de Cantavieja (Teruel) o Tortosa (Tarragona) que a los habitantes de las ciudades de Castellón o Valencia.

Figura 2. Localización del área natural Maestrazgo-Els Ports

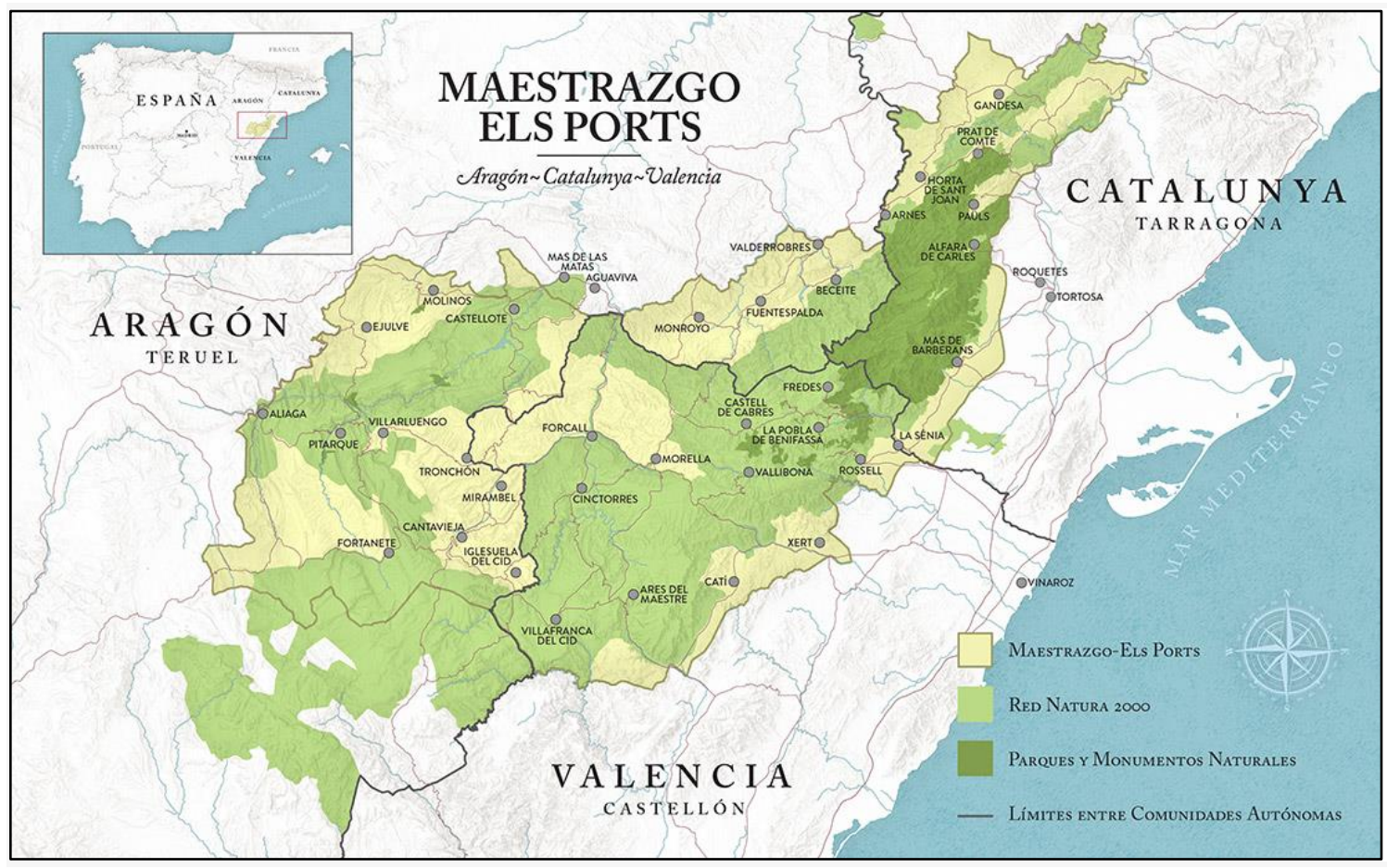

Fuente: https://www.maestrazgoports.org/ Recuperado el 04 de abril de 2020.

En lo que se refiere a sus valores naturales, a) el $58 \%$ de la región de Maestrazgo-Els Ports está incluido dentro de la Red Natura 2000, la cual incluye los parques naturales de Els Ports en Tarragona y la Tinença de Benifassà en Castelló y cuatro monumentos naturales en Teruel; b) abundan los paisajes montañosos silvestres de alto valor escénico y de conservación; c) existen poblaciones significativas de fauna llamativa como la cabra montés, buitre leonado, águila real o corzo; y d) los tres gobiernos están colaborando para traer de vuelta al quebrantahuesos, una especie altamente llamativa y considerada como 
amenazada en Europa. A estos valores naturales se suman unos pueblos históricos de singular belleza y buen estado de conservación como es el caso de Mirambel, Iglesuela, Tronchón, Villarluengo, Molinos, Cantavieja, Valderrobres y Beceite en Teruel; Morella, Villafranca, Vallibona, Ballestar, Ares de Maestre, Forcall y La Pobla de Benifassà en Castellón; y Arnes, Horta de Sant Joan, Prat de Comte y Paüls en Tarragona.

Es precisamente la combinación de grandes espacios naturales con bellos pueblos históricos lo que hace que Maestrazgo-Els Ports se pueda convertir en uno de los grandes destinos de turismo de naturaleza y cultura del mundo. Si solo fuera por naturaleza, la región no acabaría de situarse dentro de los grandes destinos globales. No puede competir con los grandes parques africanos, asiáticos y americanos. Y en lo que se refiere a fauna, le falta para llegar a los niveles de Sierra Morena, la Cordillera Cantábrica o Pirineos en la Península Ibérica. Si nos centramos exclusivamente en cultura e historia, debe competir con al menos dos docenas de comarcas en España llenas de pueblos y ciudades bellísimos, y con un centenar de otros tantos lugares en Europa. Sin embargo, cuando combinamos la naturaleza y los pueblos de la región, sí podemos pensar en un lugar que puede competir favorablemente con los mejores de mundo (Figura 3).

Figura 3. Objetivo del proyecto

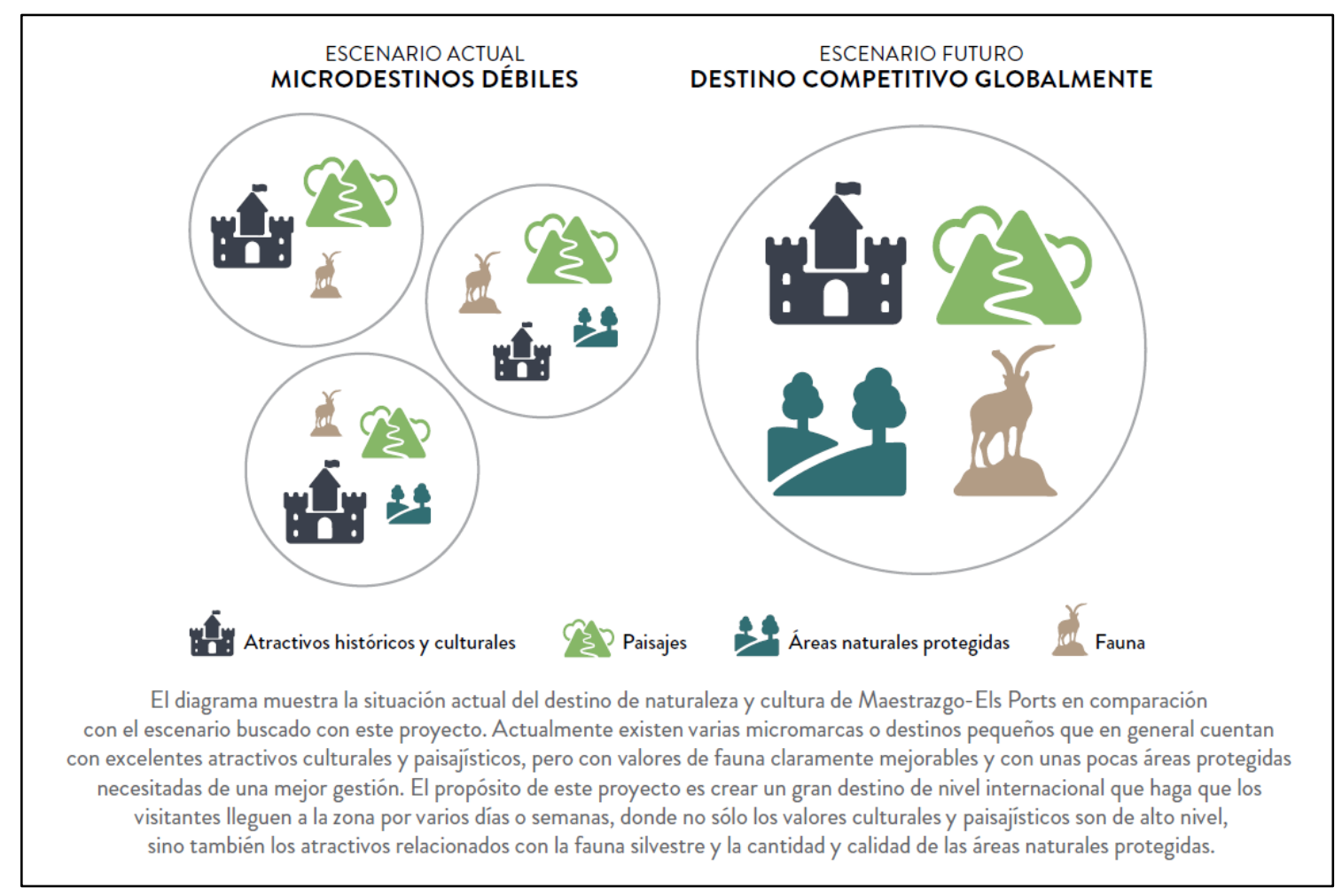

Fuente: https://www.maestrazgoports.org/ Recuperado el 04 de abril de 2020.

Una vez ubicada el área de trabajo, se comenzó a aplicar la metodología de uso combinado de comunicación y articulación. Un factor diferencial del contexto español/europeo frente al sudamericano es el mayor peso de las autoridades ambientales en el primer caso, las cuales no solo cuentan con mayor autoridad legal en términos generales, sino que -sobre todo- cuentan con mayores recursos humanos y financieros para afectar procesos públicos ambientales. Como resultado de este "hecho diferencial europeo", consideré que, a diferencia del contexto sudamericano donde las ONG tenían 
un rol de claro liderazgo en los procesos previamente descritos, en el caso español era esencial crear un espacio de liderazgo compartido con representantes de las autoridades ambientales de los tres gobiernos autonómicos. De este modo, en los primeros meses se fue conformando un grupo de trabajo conjunto, informal pero funcional compuesto por representantes de los tres gobiernos. Este grupo se ha ido consolidando y ampliando desde el año 2018 hasta la actualidad, y sigue plenamente activo. El grupo decide acciones y estrategias a seguir dentro de los intereses, oportunidades y limitaciones de cada oficina gubernamental, pero siempre con base en una visión centrada en un territorio compartido y consensuado junto al concepto de producción de naturaleza (i.e. espacios protegidos + atractivos naturales aumentados + destino turístico + empleo local). A partir de este grupo de trabajo informal, se fueron ampliando las acciones de articulación a partir de conversaciones con alcaldes, asociaciones de empresarios, representantes de la industria cinegética, ganaderos o propietarios de tierras. Este trabajo de articulación es constante y actualmente trabaja fundamentalmente en la escala local, comarcal y regional, sin haber escalado todavía al nivel nacional. En el ámbito internacional se logró obtener una donación por parte de una fundación estadounidense, la cual permite cubrir las acciones de articulación, comunicación y diagnósticos sobre la realidad regional.

Gracias a estos fondos se ha iniciado una campaña de comunicación similar a las desarrolladas en Argentina y Brasil, donde se promociona la región de Maestrazgo-Els Ports como un destino global de naturaleza y cultura, lo que incluye valorar los espacios protegidos como pilares de desarrollo local y la fauna silvestre como un activo económico, en lugar de un pasivo o molestia, como es vista en muchos casos. Esta campaña empezó a principios de 2020 con el uso de redes sociales, videos, web y una primera publicación de alta calidad visual (www.maestrazgoports.org). En lo que se refiere a la historia que se está comunicando, el "experimento español" presenta interesantes diferencias con las iniciativas de Argentina y Brasil. En primer lugar, la superficie de áreas protegidas de Maestrazgo-Els Ports es significativamente menor que en las experiencias sudamericanas, además de que los ecosistemas naturales están más fragmentados y en peor estado de conservación, especialmente en lo que se refiere a la ausencia de numerosas especies de fauna silvestre. En segundo lugar, la calidad del patrimonio cultural basado en los pueblos (el cual incluye arquitectura, arte, gastronomía, folclore e historia) es notoriamente superior en el caso español, lo que permite crear un destino netamente europeo donde la experiencia del visitante y el patrimonio a conservar y restaurar, se centran tanto en espacios naturales como en áreas humanizadas.

En tercer lugar, la infraestructura turística y de uso público es notoriamente superior en el caso español, lo que hace que no se necesite invertir tantos recursos en reforzarla y comunicarla. Esto incluye además una oferta casi abrumadora de información turística sobre la región, con el inconveniente de que se presenta a través de una multitud de productos y marcas municipales o comarcales que difícilmente pueden generar un destino global por sí mismas. En este caso, lo que se requiere es un proceso de comunicación que amplíe su escala espacial para abarcar a una región transcomunitaria, integre los aspectos culturales con los naturales en un relato contemporáneo de alcance global, a la vez que se destila y reduce el volumen de información disponible para que pueda ser asimilada por un público general.

La iniciativa Maestrazgo-Els Ports está recién dando sus primeros pasos y en los próximos años veremos si el enfoque de Producción de Naturaleza desarrollado en el Hemisferio Sur puede ser aplicado con éxito en un paisaje rural español y el tipo de ajustes necesarios para que esto suceda. Hasta este punto, el viaje ha sido apasionante. 


\section{REFERENCIAS}

Butler, T. (ed.) (2016). Tompkins Conservation 25: A Quarter Century of Work to Save the Wild. San Francisco: Tompkins Conservation. Recuperado de http://www.deepecology.org/books/25.pdf (23/03/2020).

Deinet, S., Ieronymidou, C., McRae, L., Burfield, I. J., Foppen, R. P., Collen, B., y Böhm, M. (2013). Wildlife comeback in Europe. The recovery of selected mammal and bird species. Zoological Society of London, UK.

European Environment Agency (2018). Natura 2000 Barometer. Recuperado de https://www.eea.europa.eu/data-and-maps/dashboards/natura-2000-barometer $(23 / 03 / 2020)$.

Heinonen, S. (2018). Construyendo puentes con el nivel político al renombrar nuestro trabajo como "Producción de Naturaleza". En: Jiménez Pérez, I., Producción de naturaleza: parques, rewilding y desarrollo local (pp. 65-69), Tundra.

INE (2019). España en cifras 2019. https://www.ine.es/prodyser/espa_cifras/2019/48/ $(23 / 03 / 2020)$.

Jiménez Pérez, I. (2018). Producción de naturaleza: parques, rewilding y desarrollo local. Almenara Castellón: Tundra.

Ribeiro, M. C., Martensen, A. C., Metzger, J. P., Tabarelli, M., Scarano, F., y Fortin, M. J. (2011). The Brazilian Atlantic Forest: a shrinking biodiversity hotspot. En: Zachos, E., y Habel, J. C., Biodiversity hotspots (pp. 405-434), Heidelberg. Recuperado de https://link.springer.com/chapter/10.1007/978-3-642-20992-5_21 (23/03/2020).

Zamboni, T., Di Martino, S., y Jiménez-Pérez, I. (2017). A review of a multispecies reintroduction to restore a large ecosystem: the Iberá Rewilding Program (Argentina). Perspectives in ecology and conservation, 15(4), 248-256. https://doi.org/10.1016/j.pecon.2017.10.001 